\title{
Magnetic Particles in the Human Body: A Short Review on the Effects of these Particles on the Human Health
}

\author{
Manfred Fähnle* \\ Former Member of the Max Planck Institute for Intelligent Systems, Germany
}

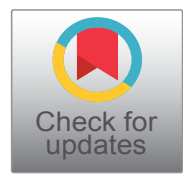

*Corresponding author: Manfred Fähnle, Former Member of the Max Planck Institute for Intelligent Systems, Stuttgart, Schönblickstraße 95, 71272 Renningen, Germany

\begin{abstract}
Magnetic particles in the human body have various effects on the human health, ranging from positive effects in the treatment of diseases, e.g., by a hyperthermia treatment, to negative effects, e.g., on Alzheimer's disease. In the present manuscript a short review is given on these effects of magnetic particles in the human body.
\end{abstract}

\section{Hyperthermia}

In reference [1] that hyperthermia treatments of human diseases are explained. It is written that by exposing diseased tissue to elevated temperature for a period of time, called hyperthermia, is a promising treatment of human diseases. Cells are susceptible to heat damage because heat is energy that disrupts cellular pathways, especially its effects on protein, especially through its effects on protein structures and its effects on protein structure and functions. Depending on the thermal does, hyperthermia induces a heat-shock to cells, leading to cell death via a series of biochemical changes within the cells. Heat also enhances the therapeutic effects of anti-cancer agents such as ionizing radiation, often producing a combined cytotoxicity that is significantly greater than that arising from either agent alone. It is observed that inhibits DNA damage to repair, rendering the cells unable to recover from the effects of ionizing radiation, culminating in cell death or senescence. Heat, however, is neither intrinsically acting to cancer-specific cells, nor are cancer cells intrinsically more sensitive to heat. On the other hand, tumor physiology has shown to provide an advantage for hyperthermia as an effective cancer therapy.
Tumors typically possess a more heterogeneous tissue structure than their normal counterparts, and have less regulated or unregulated growth. They often display chaotic vascularity, high interstitial fluid pressure, significant deposits of dense and fibrous stroma and regions chronically deprived of oxygen - all contributing to the resistance to standard therapies. Hyperthermia ameliorates these effects by increasing blood perfusion and modifying tissue structure, thereby enhancing therapy and enabling the penetration of therapeutic agents.

Clinical application of hyperthermia requires selective delivery to the tumors with an effective dose to cause a measurable effect. Certainly, a corollary of selective delivery is that the deposited to the healthy cells and tissues must be minimized to avoid cells deleterious side effects. Certainly, a corollary selective delivery is that the deposited dose to the non-cancer cells must be minimal in order to avoid cell deleterious side effects. Many strategies to deliver heat for cancer hyperthermia emerged, with varying success. The most promising strategy is to introduce magnetic nanoparticles directly to the tumors and to expose them to time-oscillating external magnetic fields. By the time-oscillating field time-oscillating magnetic hysteresis loops are generated in the magnetic nanoparticles, thereby hysteresis effects generate heat which are deposited to the surrounding tissue, thereby increasing the temperature and producing the hyperthermia effect. In order that it is possible to generate the hysteresis loops by not too high electromagnetic fields, it is important that

Citation: Fähnle M (2022) Magnetic Particles in the Human Body: A Short Review on the Effects of these Particles on the Human Health. Int J Brain Disord Treat 8:041. doi.org/10.23937/24695866/1410041

Accepted: January 06, 2022: Published: January 08, 2022

Copyright: @ 2022 Fähnle M. This is an open-access article distributed under the terms of the Creative Commons Attribution License, which permits unrestricted use, distribution, and reproduction in any medium, provided the original author and source are credited. 
magnetic nanoparticles are used which have a small magnetic anisotropy.

\section{Magnetite Nanoparticles in the Human Brain}

In reference [2] it is discussed that magnetite nanoparticles can be introduced into the human body by breathing in magnetite polluted air in human habitats, especially in areas with much traffic. After healing in these nanoparticles, they come over the lungs to the human blood. Because many of these nanoparticles are smaller than $200 \mathrm{~nm}$ in diameter, they can enter the brain directly through the olfactory bulb. In bulk magnetite the magnetostriction is rather small. In reference [3] it is shown that magnetite nanoparticles exhibit a giant magnetostriction. I now discuss the reasons for this. Both the bulk material and the nanoparticle of magnetite have above the Verwey transition temperature of $125 \mathrm{~K}$ a cubic lattice symmetry, for which the magnitude of the magnetostriction is determined [4] by the magnetostriction constants $\lambda_{100}$ and $\lambda_{111}$. The magnitudes of the magnetostriction constants are determined by the electronic properties of the system, especially by the electronic density of states at the fermi level. In reference [5] it is shown for FeGa alloys that this density of states is determined by the magnitude of the spin-orbit coupling. In reference [6] it is discussed that at surface atoms and at near surface atoms the spin-orbit coupling is much stronger than at bulk atoms, because of the reduced symmetry at these sites. Because nanoparticles have many surface or near-surface atoms, the spin-orbit coupling is large at these sites, and this has a great influence on the electronic density of states at the Fermi level and hence the magnitudes of the magnetostriction constants are much larger than their magnitudes at bulk atoms.

As discussed in section I, magnetic particles are used in the hyperthermia treatment of cancer because in timeoscillating magnetic fields they exhibit a time-oscillating hysteresis loop, by which due to hysteresis losses heat is generated and transferred to the surroundings, so that the temperature is raised, and this temperature increase is used in hyperthermia treatments. It is also discussed in section I that this requires magnetic particles with small magnetic anisotropy, because otherwise large external time-oscillating fields have to be used. Magnetite nanoparticles have large anisotropies, and therefore for the application of hyperthermia therapies other magnetic particles with small magnetic anisotropy have to be introduced in the human brain. Then we get by application of external time-oscillating magnetic fields the required heating effect. Unfortunately, the heating of the brain and the resulting increase of the temperature promotes the formation of protein plaques. These protein plaques are among other effects responsible for Alzheimer's disease. This shows that the use of magnetic particles in time-oscillating external magnetic fields have a positive effect (application for cancer therapy), but also a negative effect for people which are endangered to get Alzheimer's disease.

There is also another bad effect of the magnetite nanoparticles in the human brain. In section II it is discussed that magnetite nanoparticles have a giant magnetostriction. When applying a time-oscillating external magnetic field, then it generates timeoscillating large magnetostrictive strains, and by these also large strains on the surrounding system of neurons. By these time-oscillating strains the communications between neighboring neurons are disturbed in a time-oscillating manner, and the disturbance of the communications between neighboring neurons is also a cause for Alzheimer's disease.

In the human brain there are many billions of neurons, which communicate with each other. They send messages between different parts of the brain, and finally messages to the muscles and the organs of the human body. When the communications between these neighboring neurons is disturbed then all this is disturbed, and this contributes to Alzheimer's disease [7].

\section{Conclusion}

In the present paper it is discussed that the presence of magnetic particles in the human brain and the application of time-oscillating external magnetic fields has a very positive effect in cancer therapy, but also a negative effect because the related processes contribute to Alzheimer's disease. Therefore, it has been reflected whether in patients which are endangered to get Alzheimer's disease the hyperthermia treatment of cancer should not be replaced by conventional cancer therapies which do not have these bad effects (but which of course are inferior for cancer therapy in comparison with the hyperthermia therapy).

\section{References}

1. Dennis CL, Ivkov R (2013) Physics of heat generation using magnetic nanoparticles for hyperthermia. Int J Hyperthermia 29: 715-729.

2. Maher BA, Ahmed IAM, Karloukovski V, MacLaren DA, Foulds PG, et al. (2016) Magnetite pollution nanoparticles in the human brain. Proc Natl Acad Sci USA 113: 1079710801.

3. Balaji G, et al. (2012) Materials Science and Engineering 177: 44 .

4. Kronmüller H, Fähnle M (2003) Micromagnetism and the microstructure of ferromagnetic solids. Cambridge University Press, Cambridge, UK.

5. Wu R (2002) Origin of large magnetostriction in FeGa alloys. JAppl Phys 91: 7358.

6. Flipse CFJ, et al. (1999). European Physical Journal D9. 479.

7. Garden GA, Spada ARL (2012) Intercellular (mis) communication in neurodegenerative disease. Neuron 73: 886-901. 